\title{
Inhibition of Ocular Neovascularization by Co-Inhibition of VEGF-A and PLGF
}

\author{
Xiaochuan Huo Youxiang $\mathrm{Li}^{\mathrm{a}}$ Yuhua Jiang ${ }^{\mathrm{a}}$ Xiaoyun Sun ${ }^{\mathrm{b}}$ Lixue Gu ${ }^{\mathrm{b}}$ \\ Wenshi Guo ${ }^{b}$ Dapeng Sun ${ }^{c}$
}

aDepartment of Interventional Neuroradiology, Beijing Neurosurgical Institute, Beijing Tiantan Hospital, Capital Medical University, Beijing, ${ }^{b}$ Department of Neurosurgery, the First Affiliated Hospital of Liaoning Medical University, Jinzhou, '1B 96K Seven-Year Program, China Medical University, Shenyang, China

\section{Key Words}

Age-related macular degeneration (AMD) - Choroidal neovascularization (CNV) - Placental growth factor (PLGF) - Vascular endothelial growth factor A (VEGF-A) - Laser burn (LB) • Macrophages

\begin{abstract}
Background/Aims: Age-related macular degeneration (AMD) appears to be a disease with increasing incidence in Western countries and may develop into acquired blindness. Choroidal neovascularization (CNV) is the most frequent cause for AMD, and is commonly induced by regional inflammation. Past studies have highlighted vascular endothelial growth factor $A$ (VEGF-A) as a major trigger for CNV. However, studies on the associated angiogenic factors other than VEGF-A are lacking. Methods: Here, we used a well-established laser burn (LB)induced experimental CNV mouse model to study the molecular mechanisms underlying the development of CNV after ocular injury. We analyzed vessel density by lectin labeling. We isolated macrophages, endothelial cells and other cell types by flow cytometry, and analyzed levels of different angiogenic factors in these populations. We used antisera against VEGF-A (aVEGF) and/or antisera against placental growth factor (PLGF; aPLGF) to antagonize CNV. We used an antibody-driven toxin to selectively eliminate macrophages to evaluate the role of macrophages in CNV. We also examined expression of PLGF in macrophage subtypes. Results: The choroidal vessel density increased significantly 7 days after LB. LB increased significantly the levels of VEGF-A and PLGF in mouse eyes. Treatment with aVEGF significantly blunted increases in vessel density by LB. Treatment with aPLGF alone did not significantly reduce increases in vessel density. However, aPLGF significantly increased the inhibitory effects of aVEGF on vessel density increases. While VEGF-A was produced by endothelial cells, macrophages and other types at similar levels, PLGF seemed to be predominantly produced by macrophages. Selective macrophage depletion significantly reduced CNV. M2, but M1 macrophages produced high levels of PLGF. Conclusions: Together, our data suggest a previously unappreciated role of PLGF in coordination with VEGF-A to regulate CNV during ocular injury. Our study highlights macrophages and their production of PLGF as novel targets for CNV therapy.
\end{abstract}

Copyright (c) 2015 S. Karger AG, Basel 


\section{Introduction}

Ocular neovascularization occurs in various ocular diseases, including proliferative diabetic retinopathy, age-related macular degeneration (AMD), retinopathy of prematurity and ocular hemangioma [1-4]. Both retinal and iris neovascularization can be induced by retinal hypoxia in diseases such as diabetic retinopathy and branch retinal vein occlusion and choroidal neovascularization (CNV) [1-4]. Most CNV occurs in macular lesions at the center of the retina and directly causes a severe loss of visual acuity [1-4]. Thus, all these diseases afflict persons in all stages of life from birth through late adulthood and account for most instances of legal blindness.

Inflammatory processes are thought to be critical in generating CNV [5-7]. Many experimental and clinical studies have indicated that the vascular endothelial growth factor A (VEGF-A) family, especially VEGF-A, is essential for promoting CNV [8-12]. This growth factor also influences the production of various pro-inflammatory cytokines. However, although anti-angiogenic treatments have proven therapeutic effects with antagonist against VEGF-A, the therapeutic outcome in patients is not always satisfactory [1-12].

The VEGF family is composed of six secreted proteins: VEGF-A, VEGF-B, VEGF-C, VEGF-D, VEGF-E and placental growth factor (PLGF) [13-15]. VEGF-A-mediated angiogenesis and neovascularization have been shown to be most important for embryonic and adult vessel formation and maintenance [16-20]. PLGF may play an important role in the pathological angiogenesis, in a coordinated way with other VEGF family members. However, the exact effect of PLGF on neovascularization may be case-dependent. For example, PLGF has been reported to either promote angiogenesis and tumor growth [21], or inhibit tumor angiogenesis by forming inactive VEGF/PLGF heterodimer [22]. There are three main subtypes of VEGF receptor (VEGFR), numbered 1, 2 and 3. Also, they may be membrane-bound or soluble, depending on alternative splicing. VEGF-A binds to both VEGFR1 and VEGFR2 [15]. VEGFR2 appears to mediate almost all of the known cellular responses to VEGF. The function of VEGFR1 is less well defined, although it is thought to modulate VEGFR2 signaling [15]. Interestingly, VEGFR1 is the unique binding receptor for PLGF. VEGFR3 mediates lymphangiogenesis in response to VEGF-C and VEGF-D [15]. Angiogenesis and vascularization are also inhibited by angiostatic factors, including soluble VEGFR1, endostatin, angiostatin, etc [15].

Here, we used a well-established laser burn (LB)-induced experimental CNV mouse model to study the molecular mechanisms underlying the CNV development after ocular injury $[23,24]$. We found that the vessel density significantly increased 7 days after LB. LB significantly increased the levels of VEGF-A and PLGF in mouse eyes. Treatment with antisera against VEGF-A (aVEGF) significantly reduced increases in vessel density by LB. Treatment with antisera against PLGF (aPLGF) alone did not significantly reduce increases in vessel density. However, aPLGF significantly increased the inhibitory effects of aVEGF on vessel density increases. While VEGF-A was produced by endothelial cells, macrophages and other types at similar levels, PLGF seemed to be predominantly produced by macrophages. Selective macrophage depletion significantly reduced CNV.

\section{Materials and Methods}

Mouse manipulations

All mouse experiments were approved by the Institutional Animal Care and Use Committee at Capital Medical University (Animal Welfare Assurance). Twelve week-old female C57BL/6 mice were used in the current study. Ten mice were analyzed in each experimental condition.

\section{LB-induced CNV model}

CNV was induced by laser photocoagulation and evaluated as has been described previously [23, 24]. Briefly, laser photocoagulation (wavelength: $532 \mathrm{~nm}, 0.1 \mathrm{~s}$, spot size: $75 \mu \mathrm{m}$, power: $200 \mathrm{~mW}$ ) was performed at four spots around the optic disc of one eye/mouse. 


\section{Choroidal vessel density quantification}

One week after LB, the mice were perfused with $1 \mathrm{ml}$ PBS containing $50 \mathrm{mg} / \mathrm{ml}$ Texas Red labeled Lycopersicon Esculentum (Tomato) Lectin (Vector Labs, Burlingame, CA, USA), and the eyes were removed. The entire retina was carefully dissected from the eyecup and flat-mounted on an aqua-mount with the sclera facing downward and the choroid facing upward. Fluorescent images of the choroidal flat mounts were taken. The largest hyperfluorescent area corresponding to a photocoagulation spot for each eye was evaluated using NIH ImageJ software and subjected to quantitative analyses.

\section{Intravitreal Injections}

Intravitreal injections were performed under a surgical microscope. Animals were anaesthetized with the $2.5 \%$ anesthesia isofluorane. Pupils were dilated using $1 \%$ tropicamide and $2.5 \%$ phenylephrine (SigmaAldrich, St. Louis, MO, USA). Viscotears Liquid Gel and a microscope coverslip were used to improve the visibility of the fundus. A 33 -gauge needle (Sigma-Aldrich) was inserted from the limbus with a $45^{\circ}$ injection angle into the vitreous. The direction and location of the needle was monitored through the microscope. A volume of $2 \mu \mathrm{l}$ antisera or reagents was injected using a repeating dispenser (PB-600-1; Hamilton Bonaduz, Reno, NV, USA). All procedures were conducted by the same surgeon.

Anti-VEGFa (aVEGF) and anti-PLGF (aPLGF) antisera were purchased from Sigma-Aldrich (USA) and were injected into mice at 1,3 and 5 days after induction of LB at a dose of $10 \mu \mathrm{g}$ in $2 \mu$ l Phosphate Buffered Saline (PBS, Sigma-Aldrich). Sham mice received PBS of same volume.

Saporin-conjugated anti-CD11b antibody $(20 \mu \mathrm{g} / 2 \mu \mathrm{l}$; Advanced Targeting Systems, San Diego, CA, USA) against the pan-macrophage surface marker CD11b was injected once at 1 day after LB to eliminate macrophages [25], while the control group received injection of same volume of IgG at the same time.

Flow cytometry

F4/80 and CD31-based cell analysis and sorting were performed by flow cytometry, after the cells were labeled with PEcy7-conjugated anti-F4/80 and APC-conjugated CD31 antibodies (Becton-Dickinson Biosciences, San Jose, CA, USA), or with anti-F4/80 and Pacific blue-conjugated anti-CD206 (BectonDickinson Biosciences). Flow cytometry was performed using a FACSAria (Becton-Dickinson Biosciences) flow cytometer. Data were analyzed and quantified using Flowjo software (Flowjo LLC, Ashland, OR, USA).

\section{RT-qPCR}

RNA was extracted from cells with RNeasy kit (Qiagen, Hilden, Germany) and used for cDNA synthesis. Quantitative PCR (RT-qPCR) were performed in duplicates with QuantiTect SYBR Green PCR Kit (Qiagen). All primers were purchased from Qiagen. Data were collected and analyzed with the Rotorgene software accompanying the PCR machine, using 2- $\Delta \Delta \mathrm{Ct}$ method for quantification of the relative mRNA expression levels. Values of genes were first normalized against $\alpha$-tubulin, and then compared to controls.

\section{ELISA}

The concentration of VEGF-A, VEGF-B, VEGF-C, VEGF-D, VEGF-E and PLGF in the cells was determined by corresponding ELISA Kit (R\&D System, Los Angeles, CA, USA). ELISAs were performed according to the instructions of the manufacturer. Briefly, the collected condition media was added to a well coated with primary antibody, and then immunosorbented by biotinylated primary antibody at room temperature for 2 hours. The color development catalyzed by horseradish peroxidase was terminated with $2.5 \mathrm{~mol} / \mathrm{l}$ sulfuric acid and the absorption was measured at $450 \mathrm{~nm}$. The protein concentration was determined by comparing the relative absorbance of the samples with the standards.

\section{Statistical analysis}

All statistical analyses were carried out using the SPSS 19.0 statistical software package. All data were statistically analyzed using one-way ANOVA with a Bonferoni Correction. All values are depicted as mean \pm standard deviation and are considered significant if $\mathrm{p}<0.05$. 


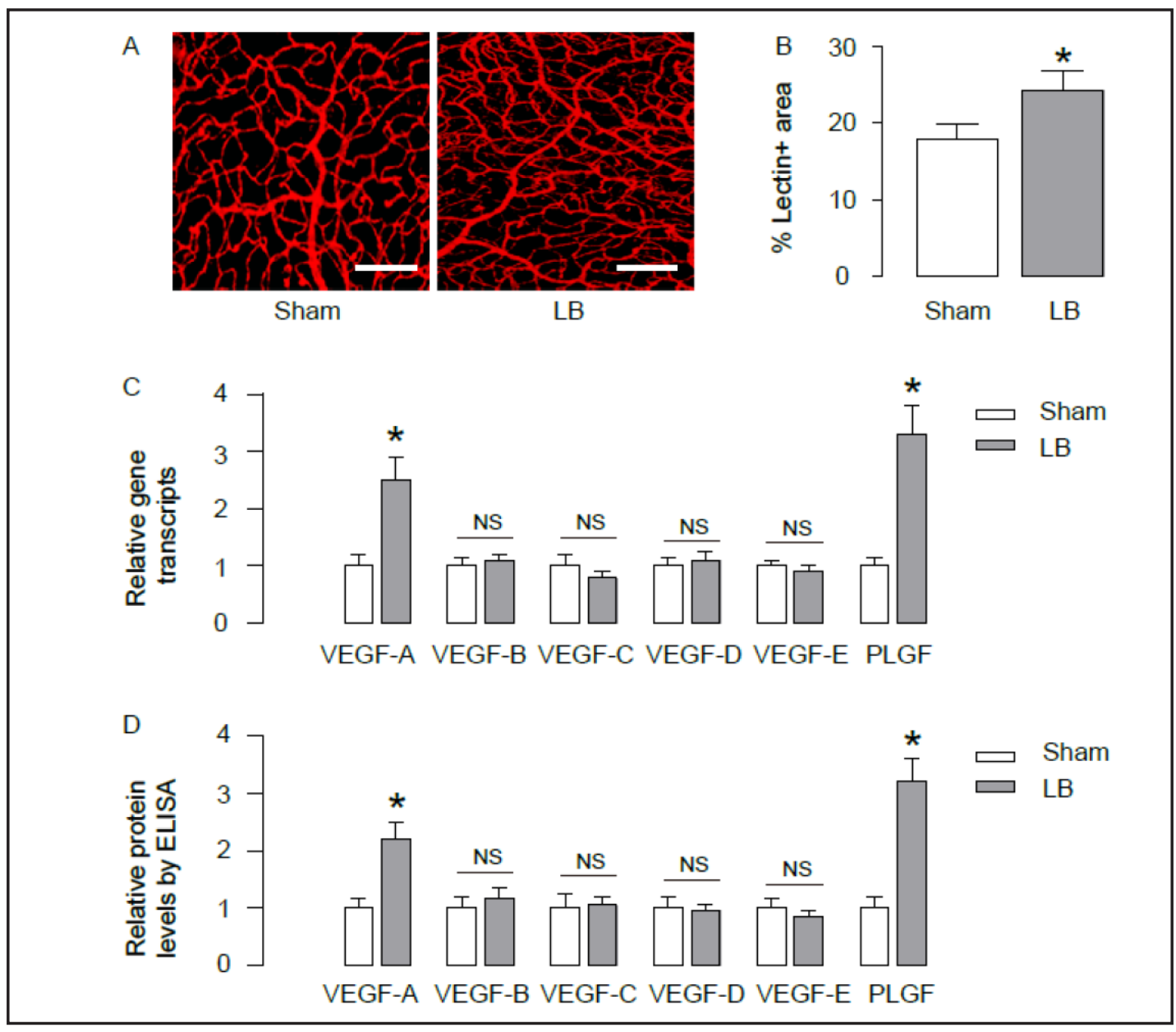

Fig. 1. VEGF-A and PLGF levels increased in mouse eyes after LB. Seven days after laser burn (LB), the ocular vessel density was examined by lectin labeling. (A-B) Vessel density was examined, shown by representative images (A) and by quantification (B). (C-D) The levels of VEGF family members in mouse eyes were examined by RT-qPCR (C) and by ELISA (D). * ${ }^{*}<0.05$. NS: non-significant. N=10. Statistics: one-way ANOVA with a Bonferoni Correction. Scale bars are $50 \mu \mathrm{m}$.

\section{Results}

\section{VEGF-A and PLGF levels increased in mouse eyes after $L B$}

We used a well-established laser burn (LB)-induced experimental CNV mouse model to study the molecular mechanisms underlying the CNV development after ocular injury. Seven days after $\mathrm{LV}$, the choroidal vessel density was examined by lectin labeling. We found that the LB-treated mice had significantly higher choroidal vessel density, compared to sham-treated mice (Fig. 1A-B). Thus, this model is proper for your further analyses.

Then we examined the levels of VEGF family members in mouse eyes by RT-qPCR (Fig. 1C) and by ELISA (Fig. 1D). While we did not detect differences in levels of VEGF-B, VEGF-C, VEGF-D and VEGF-E, significantly higher levels of VEGF-A and PLGF were detected in LBtreated mouse eyes, compared to controls (Fig. 1C-D). These data suggest that besides an established role of VEGF-A in the CNV, PLGF may be also involved in the pathological process.

Co-inhibition of PLGF significantly substantiated the anti-CNV effects of VEGF-A inhibition

Thus, we intravitreally injected antisera against VEGF-A (aVEGF) and/or antisera against PLGF (aPLGF) at 1, 3 and 5 days after LB to antagonize CNV, and analyzed vessel density at day 7 (Fig. 2A). We found that treatment with aVEGF significantly reduced increases in KARGER 
A

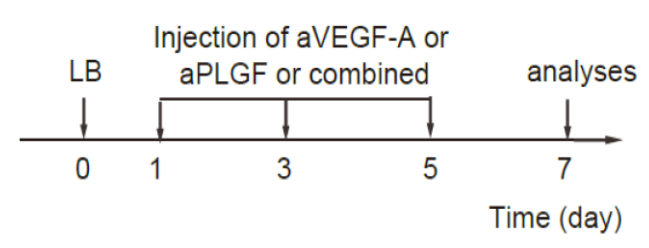

B

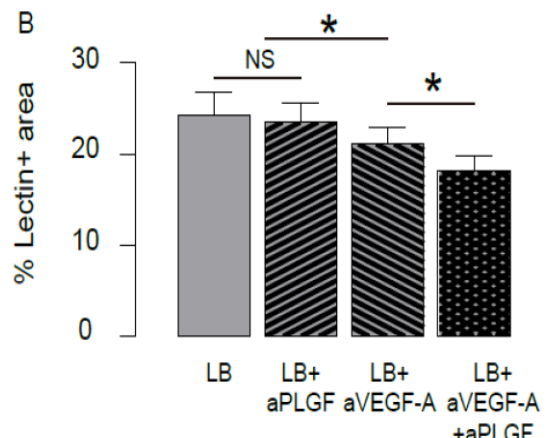

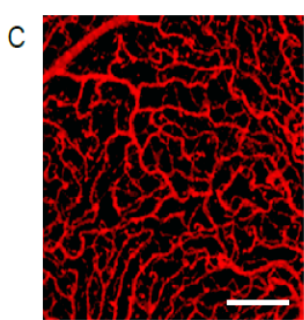

LB

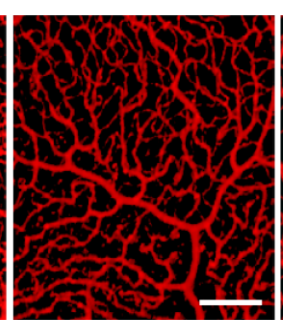

$\mathrm{LB}+$

aPLGF

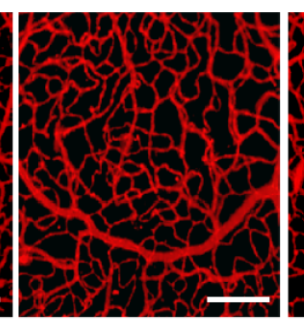

$L B+$

aVEGF-A
(C) 2015 S. Karger AG, Basel

www.karger.com/cpb

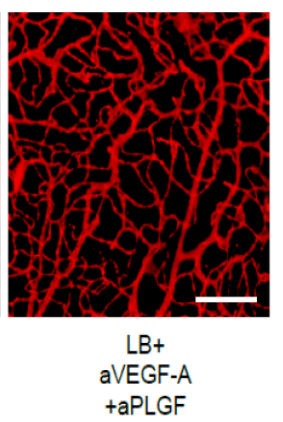

Fig. 2. Co-inhibition of PLGF significantly substantiated the anti-CNV effects of VEGF-A inhibition. (A) Intravitreal injections of antisera against VEGF-A (aVEGF) and/or antisera against PLGF (aPLGF) at 1, 3 and 5 days after LB were performed to antagonize CNV. (B-C) Vessel density was examined, shown by quantification (B) and by representative images (C). ${ }^{*} p<0.05$. NS: non-significant. $N=10$. Statistics: one-way ANOVA with a Bonferoni Correction. Scale bars are $50 \mu \mathrm{m}$.

vessel density by LB (Fig. 2B-C). Treatment with aPLGF alone did not significantly reduce increases in vessel density (Fig. 2B-C). However, aPLGF significantly increased the inhibitory effects of aVEGF on vessel density increases (Fig. 2B-C). Thus, co-inhibition of PLGF seems to substantiate the anti-CNV effects of VEGF-A inhibition, which has not been appreciated in the previous studies.

\section{PLGF was mainly produced by macrophages after $L B$}

Since macrophages have been recently reported as a major source of PLGF in other disease models [26-30], we thus isolated macrophages (based on surface marker F4/80), endothelial cells (based on surface marker CD31) and other cell types (F4/80-CD31- cells) in mouse eyes by flow cytometry (Fig. 3A), and analyzed levels of VEGF-A and PLGF in these populations by RT-qPCR (Fig. 3B) and by ELISA (Fig. 3C). We found that while VEGF-A was produced by endothelial cells, macrophages and other types at similar levels (no significant difference, Fig. 3B-C), PLGF seemed to be predominantly produced by macrophages (Fig. $3 \mathrm{~B}-\mathrm{C}$ ). These data suggest that unlike VEGF-A, PLGF is mainly produced by macrophages after LB.

\section{Selective macrophage depletion significantly reduced CNV}

Since PLGF is mainly produced by macrophages after LB, and since co-inhibition of PLGF substantiates the anti-CNV effects of VEGF-A inhibition, we hypothesize that selective macrophage depletion may significantly reduce CNV. To prove it, we used an antibodydriven toxin to selectively eliminate macrophages to examine its effects on CNV. CD11b is a specific marker for macrophages. A saporin-conjugated antibody against CD11b has been shown to efficiently eliminate macrophages [25]. We thus did single intravitreal injection of saporin-conjugated antibody against CD11b, or control solution, at 1 day after LB (Fig. 4A). 


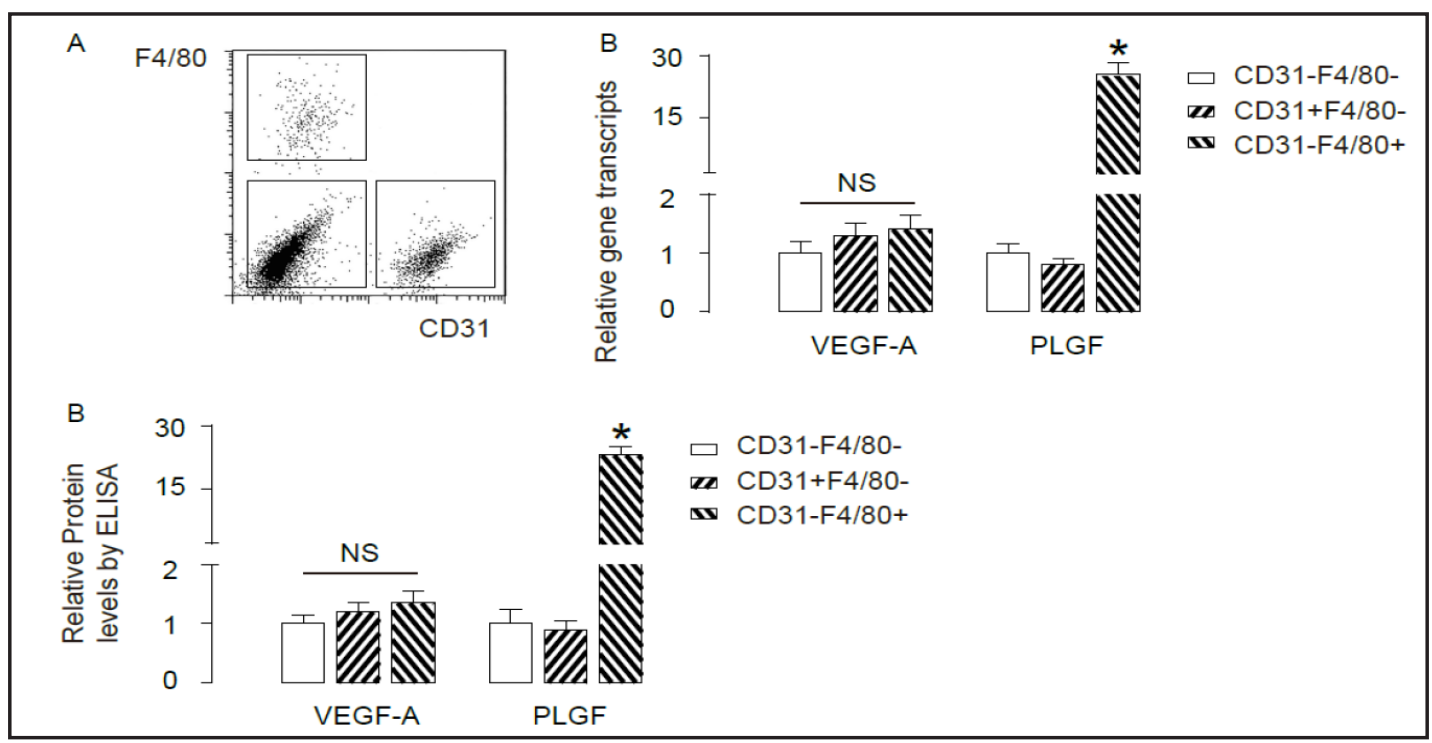

Fig. 3. PLGF was mainly produced by macrophages after LB. (A) Macrophages (based on F4/80+), endothelial cells (based on CD31+) and other cell types (F4/80-CD31- cells) in the eyes were separated by flow cytometry, shown by representative flow chart. (B-C) The levels of VEGF-A and PLGF in these populations were analyzed by RT-qPCR (B) and by ELISA (C). ${ }^{*} p<0.05$. NS: non-significant. N=10. Statistics: one-way ANOVA with a Bonferoni Correction.

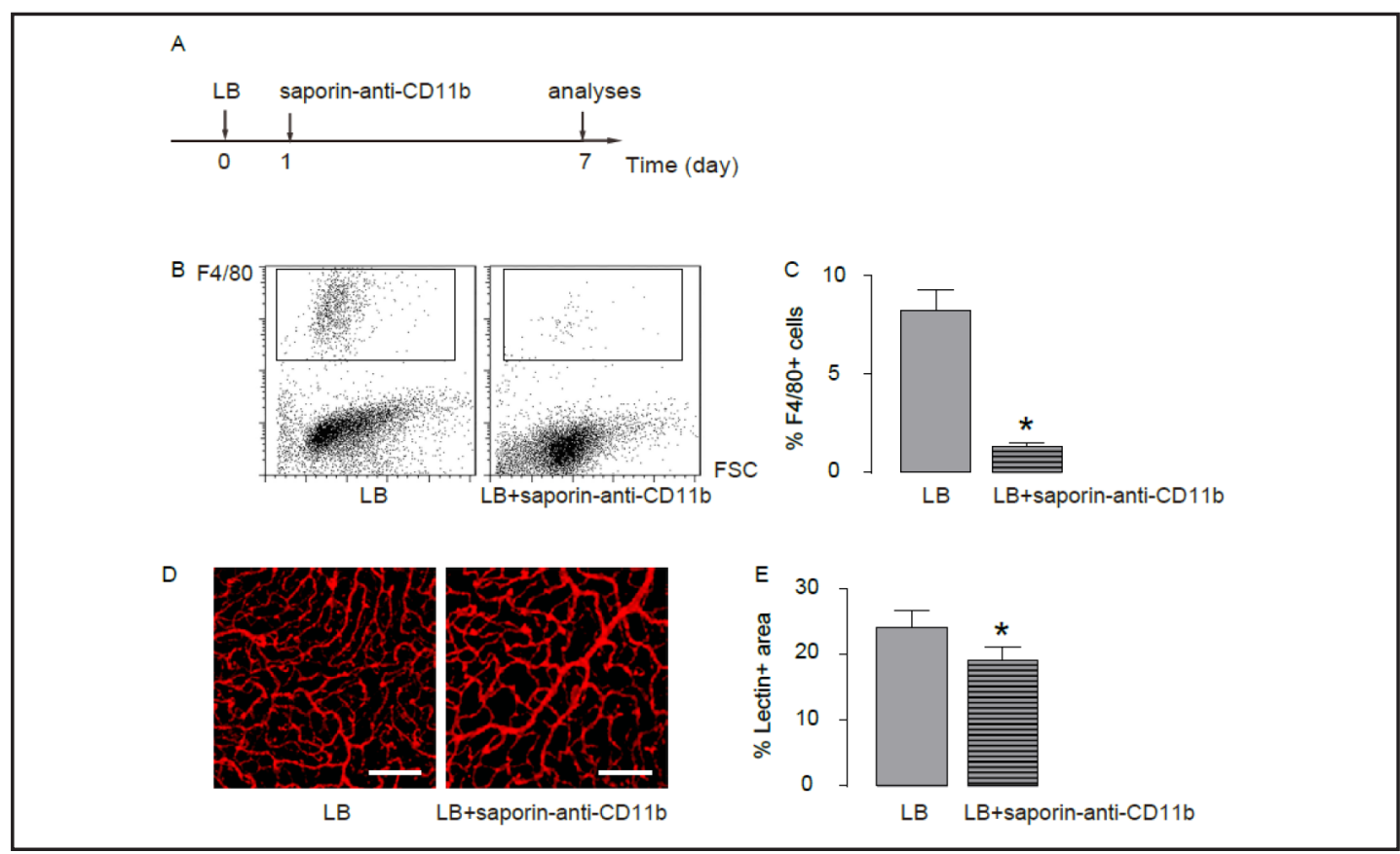

Fig. 4. Selective macrophage depletion significantly reduced CNV. (A) Single intravitreal injection of saporinconjugated antibody against CD11b (saporin-anti-CD11b) to selectively eliminate macrophages at 1 day after LB. (B-C) The elimination of macrophages was confirmed by examination of macrophage percentage by flow cytometry, shown by representative flow charts (B) and by quantification (C). (D-E) Vessel density was examined, shown by representative images (D) and by quantification (E). ${ }^{*} \mathrm{p}<0.05$. NS: non-significant. $\mathrm{N}=10$. Statistics: one-way ANOVA with a Bonferoni Correction. Scale bars are $50 \mu \mathrm{m}$.

The elimination of macrophages was confirmed by examination of macrophage percentage by flow cytometry (Fig. 4B), showing a significant decrease (85\% decrease, Fig. 4C). Further, macrophage depletion significantly reduced the increases in vessel density by LB (Fig. 4D-E). 


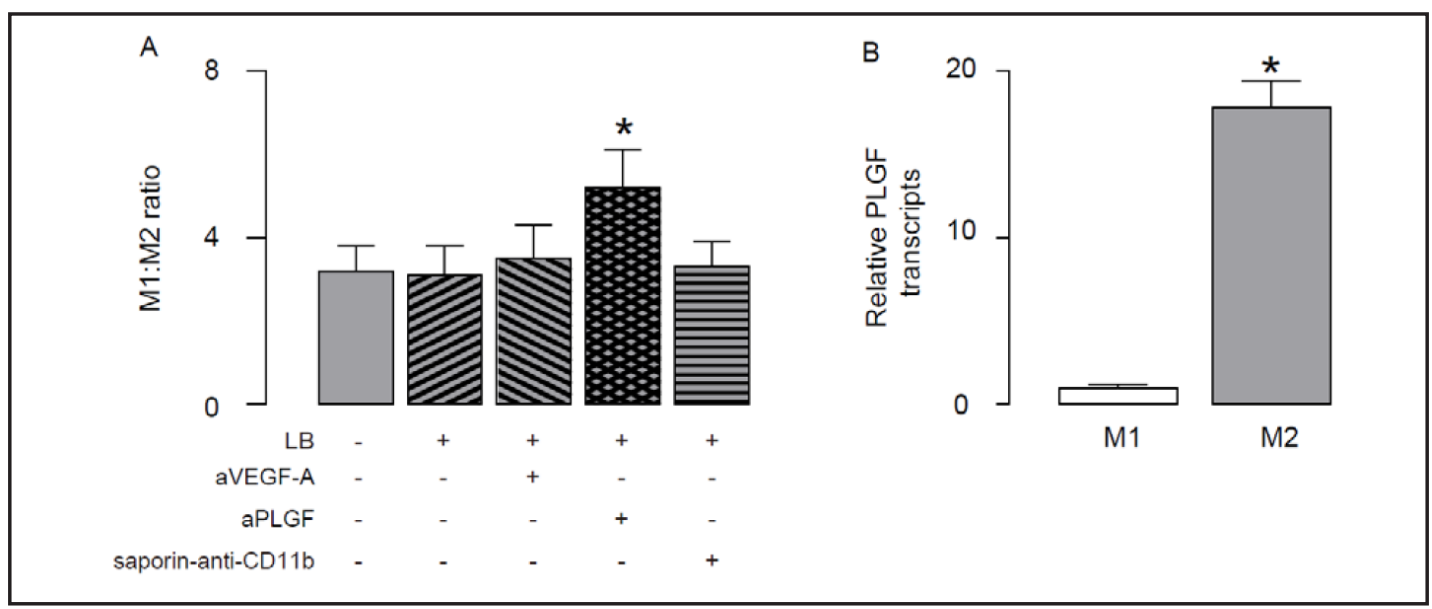

Fig. 5. PLGF was mainly produced by M2 macrophages. (A) In order to figure out the production of PLGF by macrophage subtypes, we used a M2-specific cell surface marker CD206 to label cells in different conditions, and found that neither anti-VEGF-A treatment, nor macrophage ablation affected the ratio of M1 to M2 macrophages. However, anti-PLGF treatment significantly increased the M1:M2 ratio, suggesting that M2 macrophage polarization may be inhibited by PLGF antagonizing. (B) Significant higher levels of PLGF transcripts were detected in M2 macrophages, compared to M1. ${ }^{*} \mathrm{p}<0.05$. NS: non-significant. N=10. Statistics: one-way ANOVA with a Bonferoni Correction. Scale bars are $50 \mu \mathrm{m}$.

Fig. 6. Schematic of the model. Macrophages produce PLGF and VEGF-A, and endothelial cells and other cells produce VEGF-A; VEGF-A triggers CNV, which could be substantialized by PLGF.

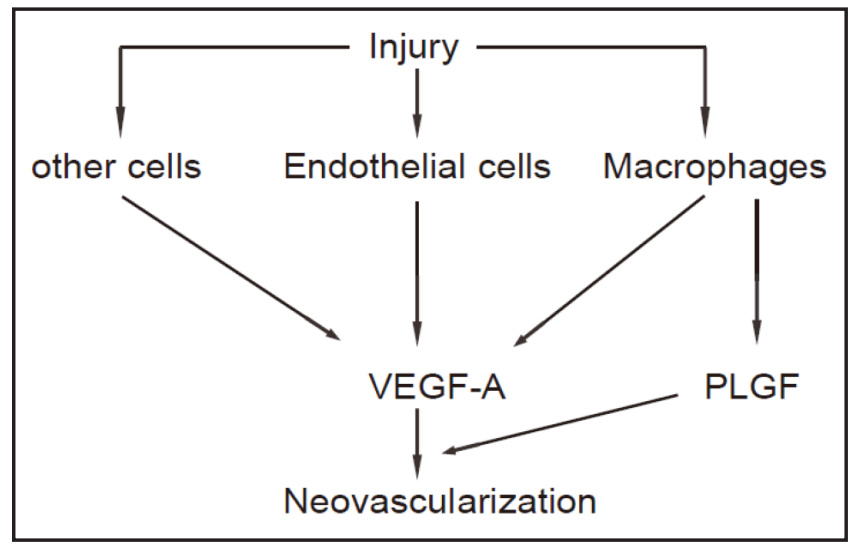

PLGF was mainly produced by M2 macrophages

In order to figure out the production of PLGF by macrophage subtypes, we used a M2-specific cell surface marker CD206 to label cells in different conditions, and found that neither anti-VEGF-A treatment, nor macrophage ablation affected the ratio of M1 to M2 macrophages (Fig. 5A). However, anti-PLGF treatment significantly increased the M1:M2 ratio, suggesting that M2 macrophage polarization may be inhibited by PLGF antagonizing. Moreover, significant higher levels of PLGF transcripts were detected in M2 macrophages, compared to M1 (Fig. 5B). Together, these data suggest that PLGF is mainly produced by M2 macrophages. Our study demonstrates a model in that after injury, macrophages produce PLGF and VEGF-A, and endothelial cells and other cells produce VEGF-A; VEGF-A triggers CNV, which could be substantialized by PLGF (Fig. 6).

\section{Discussion}

Ocular neovascularization can be induced by retinal hypoxia in diseases such as diabetic retinopathy and branch retinal vein occlusion and CNV [1-4]. Most CNV occurs in macular lesions at the center of the retina and directly causes a severe loss of visual acuity 
[1-4]. Inflammation-induced VEGF-A production is essential for development of CNV [5-12]. However, although anti-VEGF-A treatments have proven therapeutic effects, the outcome remains unsatisfactory.

The VEGF family is composed of six secreted proteins, among which PLGF may play an important role in the pathological angiogenesis $[20,31]$, in a coordinated way with other VEGF family members. However, the exact effect of PLGF on neovascularization may be casedependent.

Laser burn has been recognized as a potential trigger for CNV [32]. Here, we used a wellestablished laser burn (LB)-induced experimental CNV mouse model to study the molecular mechanisms underlying the CNV development after ocular injury [23, 24]. We validated the model by examination of vessel density at 7 days after LB. We used lectin labeling, rather than an endothelial cell marker, e.g. CD31, CD106 and CD144 [13-15, 33-35], since lectin likely labels functional vessels and appears to be a better choice for analyzing the functionality of the newly formed vessels [36].

Interestingly, we found that LB significantly increased the levels of VEGF-A and PLGF, but not other VEGF family members in mouse eyes. Moreover, treatment with aVEGF but not aPLGF significantly reduced increases in vessel density by LB. However, aPLGF significantly increased the inhibitory effects of aVEGF on vessel density increases. Since PLGF and VEGF-A share a same receptor, VEGFR1 [13-15], it may be expected that PLGF substantializes the effects of VEGF-A either by modulating VEGFR1-mediated downstream signaling, or by forming heterodimer with VEGF-A to modify the ligands $[21,22]$. These mechanisms may be conducted through ligand (with different homodimers or heterodimer) competition for receptor (VEGFR1) binding [21, 22].

Next, we found that VEGF-A was produced by endothelial cells, macrophages and other types at similar levels, PLGF seemed to be predominantly produced by macrophages. These data suggest that macrophages may be the unique source for PLGF in the eyes after injury, while VEGF-A are from multiple sources. Specially, the VEGF-A in CD31-F4/80- cells may be produced by mesenchymal cells [13-15]. In order to confirm the role of PLGF, we selectively eliminated macrophages by a saporin-conjugated antibody against CD11b [25]. Saporin is a ribosome-inactivating protein from seeds of the plant Saponaria officinalis [25]. Saporin is safe for laboratory use under normal safety conditions and the LD50 in mice is $4 \mathrm{mg} /$ kg. Moreover, Saporin does not have a method of cell entry on its own [25]. Thus, CD11b antibody will direct entrance of Saporin into macrophages to selectively kill them [25]. We found that macrophage depletion significantly reduced the increases in vessel density by LB, which confirmed our hypothesis. However, besides monocytes, CD11b is also expressed by dendritic cells and can be upregulated on activated cells irrespective of their naive expression status. Thus, CD11b-driven Saporin may also eliminate some dendritic cells. Future experiments may be designed to examine the effects of dendritic cell ablation on the interpretation of the results.

According to literature [26-30], the PLGF may be predominantly secreted by a subpopulation of macrophages called M2 macrophages, which are distinct from the classical M1 macrophages. We confirmed it in the current study.

To summarize, our study shows a model in that after injury, macrophages produce PLGF and VEGF-A, and endothelial cells and other cells produce VEGF-A; VEGF-A triggers CNV, which could be substantialized by PLGF.

\section{Disclosure Statement}

The authors have declared that no competing interests exist. 


\section{Acknowledgement}

This study was supported by Natural Science Foundation of China (No. 81171111) and Natural Science Foundation of Liaoning Province of China (No. 2013022016).

\section{Reference}

$>1$

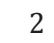

$>3$

$>4$

$>5$

$>6$

$>7$

Campochiaro PA: Ocular neovascularization. J Mol Med (Berl) 2013;91:311-321.

Cai X, Sezate SA, McGinnis JF: Neovascularization: Ocular diseases, animal models and therapies. Adv Exp Med Biol 2012;723:245-252.

Bradley J, Ju M, Robinson GS: Combination therapy for the treatment of ocular neovascularization. Angiogenesis 2007;10:141-148.

Campochiaro PA: Gene therapy for ocular neovascularization. Curr Gene Ther 2007;7:25-33.

Wolf A, Thurau S, Kook D, Hoeing A, Priglinger S, Kampik A, Haritoglou C: Intravitreal bevacizumab for choroidal neovascularization secondary to inflammation. Acta Ophthalmol 2010;88:e295-296.

Tatar O, Adam A, Shinoda K, Kaiserling E, Boeyden V, Claes C, Eckardt C, Eckert T, Pertile G, Scharioth GB, Yoeruek E, Szurman P, Bartz-Schmidt KU, Grisanti S: Early effects of intravitreal triamcinolone acetonide on inflammation and proliferation in human choroidal neovascularization. Arch Ophthalmol 2009;127:275281.

Tatar O, Yoeruek E, Szurman P, Bartz-Schmidt KU, Tubingen Bevacizumab Study G, Adam A, Shinoda K, Eckardt C, Boeyden V, Claes C, Pertile G, Scharioth GB, Grisanti S: Effect of bevacizumab on inflammation and proliferation in human choroidal neovascularization. Arch Ophthalmol 2008;126:782-790. Heier JS, Boyer DS, Ciulla TA, Ferrone PJ, Jumper JM, Gentile RC, Kotlovker D, Chung CY, Kim RY, Group FS: Ranibizumab combined with verteporfin photodynamic therapy in neovascular age-related macular degeneration: Year 1 results of the focus study. Arch Ophthalmol 2006;124:1532-1542.

Pieramici DJ, Avery RL: Ranibizumab: Treatment in patients with neovascular age-related macular degeneration. Expert Opin Biol Ther 2006;6:1237-1245.

Ferrara N, Damico L, Shams N, Lowman H, Kim R: Development of ranibizumab, an anti-vascular endothelial growth factor antigen binding fragment, as therapy for neovascular age-related macular degeneration. Retina 2006;26:859-870.

Brown DM, Kaiser PK, Michels M, Soubrane G, Heier JS, Kim RY, Sy JP, Schneider S, Group AS: Ranibizumab versus verteporfin for neovascular age-related macular degeneration. N Engl J Med 2006;355:1432-1444. Rosenfeld PJ, Brown DM, Heier JS, Boyer DS, Kaiser PK, Chung CY, Kim RY, Group MS: Ranibizumab for neovascular age-related macular degeneration. N Engl J Med 2006;355:1419-1431.

Xiao X, Prasadan K, Guo P, El-Gohary Y, Fischbach S, Wiersch J, Gaffar I, Shiota C, Gittes GK: Pancreatic duct cells as a source of vegf in mice. Diabetologia 2014;57:991-1000.

Xiao X, Guo P, Chen Z, El-Gohary Y, Wiersch J, Gaffar I, Prasadan K, Shiota C, Gittes GK: Hypoglycemia reduces vascular endothelial growth factor a production by pancreatic beta cells as a regulator of beta cell mass. J Biol Chem 2013;288:8636-8646.

Ferrara N, Gerber HP, LeCouter J: The biology of vegf and its receptors. Nat Med 2003;9:669-676. Ma J, Sawai H, Ochi N, Matsuo Y, Xu D, Yasuda A, Takahashi H, Wakasugi T, Takeyama H: Pten regulates angiogenesis through pi3k/akt/vegf signaling pathway in human pancreatic cancer cells. Mol Cell Biochem 2009;331:161-171.

Cabebe E, Fisher GA: Clinical trials of vegf receptor tyrosine kinase inhibitors in pancreatic cancer. Expert Opin Investig Drugs 2007;16:467-476.

Hotz HG, Hines OJ, Masood R, Hotz B, Foitzik T, Buhr HJ, Gill PS, Reber HA: Vegf antisense therapy inhibits tumor growth and improves survival in experimental pancreatic cancer. Surgery 2005;137:192-199.

Mao D, Zhang Y, Lu H, Zhang H: Molecular basis underlying inhibition of metastasis of gastric cancer by anti-vegfa treatment. Tumour Biol 2014;35:8217-8223.

Zhou X, Qi Y: Plgf inhibition impairs metastasis of larynx carcinoma through mmp3 downregulation. Tumour Biol 2014;35:9381-9386. 
21 Carmeliet P, Moons L, Luttun A, Vincenti V, Compernolle V, De Mol M, Wu Y, Bono F, Devy L, Beck H, Scholz D, Acker T, DiPalma T, Dewerchin M, Noel A, Stalmans I, Barra A, Blacher S, Vandendriessche T, Ponten A, Eriksson U, Plate KH, Foidart JM, Schaper W, Charnock-Jones DS, Hicklin DJ, Herbert JM, Collen D, Persico MG: Synergism between vascular endothelial growth factor and placental growth factor contributes to angiogenesis and plasma extravasation in pathological conditions. Nat Med 2001;7:575-583.

22 Eriksson A, Cao R, Pawliuk R, Berg SM, Tsang M, Zhou D, Fleet C, Tritsaris K, Dissing S, Leboulch P, Cao Y: Placenta growth factor-1 antagonizes vegf-induced angiogenesis and tumor growth by the formation of functionally inactive plgf-1/vegf heterodimers. Cancer Cell 2002;1:99-108.

23 Krzystolik MG, Afshari MA, Adamis AP, Gaudreault J, Gragoudas ES, Michaud NA, Li W, Connolly E, O'Neill CA, Miller JW: Prevention of experimental choroidal neovascularization with intravitreal anti-vascular endothelial growth factor antibody fragment. Arch Ophthalmol 2002;120:338-346.

24 Hasegawa E, Oshima Y, Takeda A, Saeki K, Yoshida H, Sonoda KH, Ishibashi T: Il-27 inhibits pathophysiological intraocular neovascularization due to laser burn. J Leukoc Biol 2012;91:267-273.

25 Kanai T, Uraushihara K, Totsuka T, Nemoto Y, Fujii R, Kawamura T, Makita S, Sawada D, Yagita H, Okumura $\mathrm{K}$, Watanabe M: Ameliorating effect of saporin-conjugated anti-cd11b monoclonal antibody in a murine t-cell-mediated chronic colitis. J Gastroenterol Hepatol 2006;21:1136-1142.

-26 Jetten N, Verbruggen S, Gijbels MJ, Post MJ, De Winther MP, Donners MM: Anti-inflammatory m2, but not pro-inflammatory $\mathrm{m} 1$ macrophages promote angiogenesis in vivo. Angiogenesis 2014;17:109-118.

-27 Kerber M, Reiss Y, Wickersheim A, Jugold M, Kiessling F, Heil M, Tchaikovski V, Waltenberger J, Shibuya M, Plate KH, Machein MR: Flt-1 signaling in macrophages promotes glioma growth in vivo. Cancer Res 2008;68:7342-7351.

28 Shen J, Xie B, Dong A, Swaim M, Hackett SF, Campochiaro PA: In vivo immunostaining demonstrates macrophages associate with growing and regressing vessels. Invest Ophthalmol Vis Sci 2007;48:43354341.

-29 Adini A, Kornaga T, Firoozbakht F, Benjamin LE: Placental growth factor is a survival factor for tumor endothelial cells and macrophages. Cancer Res 2002;62:2749-2752.

-30 Xiao X, Gaffar I, Guo P, Wiersch J, Fischbach S, Peirish L, Song Z, El-Gohary Y, Prasadan K, Shiota C, Gittes GK: M2 macrophages promote beta-cell proliferation by up-regulation of smad7. Proc Natl Acad Sci U S A 2014;111:E1211-1220.

-31 Chen Y, Jiang T, Mao A, Xu J: Esophageal cancer stem cells express plgf to increase cancer invasion through mmp9 activation. Tumour Biol 2014;35:12749-12755.

-32 Kaiser RS, Berger JW, Maguire MG, Ho AC, Javornik NB, Choroidal Neovascularization Prevention Trial Study G: Laser burn intensity and the risk for choroidal neovascularization in the cnvpt fellow eye study. Arch Ophthalmol 2001;119:826-832.

33 Gregg AJ, Schenkel AR: Cloning and structural analysis of equine platelet endothelial cell adhesion molecule (pecam, cd31) and vascular cell adhesion molecule-1 (vcam-1, cd106). Veterinary immunology and immunopathology 2008;122:295-308.

-34 Lechleitner S, Gille J, Johnson DR, Petzelbauer P: Interferon enhances tumor necrosis factor-induced vascular cell adhesion molecule 1 (cd106) expression in human endothelial cells by an interferon-related factor 1-dependent pathway. J Exp Med 1998;187:2023-2030.

- 35 Halama T, Staffler G, Hoch S, Stockinger H, Wolff K, Petzelbauer P: Vascular-endothelial cadherin (cd144)but not pecam-1 (cd31)-based cell-to-cell contacts convey the maintenance of a quiescent endothelial monolayer. Int Arch Allergy Immunol 1999;120:237-244.

-36 Zheng H, Shen CJ, Qiu FY, Zhao YB, Fu GS: Stromal cell-derived factor 1alpha reduces senescence of endothelial progenitor subpopulation in lectin-binding and dildl-uptaking cell through telomerase activation and telomere elongation. J Cell Physiol 2010;223:757-763. 